\title{
Valoración económica del Área de Conservación Regional Vilacota Maure: Servicios ecosistémicos priorizados al extremo sur del Perú
}

\author{
Economic valuation of the Vilacota Maure Regional Conservation Area: Priority ecosystem \\ services to the extreme south of Peru
}

\author{
1,2,a Marco Navarro \\ ${ }^{2, \mathrm{~b}}$ Claudio Guillen \\ ${ }^{1, c}$ Leónidas Limache
}

\begin{abstract}
ARTÍCULO ORIGINAL
${ }^{1}$ Universidad Latinoamericana CIMA. Tacna,Perú. E-mail:mnavarro@cima.edu.pe ${ }^{2}$ El Reto de la Conservación SAC. Tacna, Perú.

aRCID: 0000-0003-0516-5990

'ORCID: 0000-0002-2752-1960

'ORCID: 0000-0001-87126834
\end{abstract}

Palabras clave: Economía ecológica, ecosistemas altoandinos, forraje ganadero, recurso hídrico, servicios ecosistémicos.

Keywords: Ecological economics, high Andean ecosystems, livestock fodder, water resources, ecosystem services.

Información adicional

Presentado: 21/09/2020

Aprobado: 21/12/2020

\section{RESUMEN}

A pesar de la importancia que tienen los bienes y servicios ecosistémicos en la economía mundial, en la gestión del Área de Conservación Regional Vilacota Maure en el departamento de Tacna aún se mantienen desfasados paradigmas de uso irracional de recursos y de sobreexplotación, manifestándose en grandes presiones y amenazas a los ecosistemas, contrarias a sus objetivos de conservación. Es por ello que en una reunión de expertos (focus group), se priorizó valorizar económicamente a tres servicios ecosistémicos que representan mejor su importancia: provisión de recurso hídrico, el secuestro de carbono y provisión de recursos genéticos o forraje ganadero. Para la determinación de la valoración económica de estos servicios se aplicó el método preferencias reveladas y de precios de mercado, resultando $\$ 1749$ 548.65, \$ 454938.55 y $\$ 1461$ 388.35 respectivamente. Asimismo, también se valorizó en función a su potencial de desarrollo a través del método de preferencias reveladas y respectivamente en base a costos evitados, precios de mercado y función de producción, resultando los valores de $\$ 3844682.73$, \$ 454938.55 y \$ 7254869.32 respectivamente.

ABSTRACT
Despite the importance of ecosystem goods and services in the world
economy, the management of the Vilacota Maure Regional Conservation
Area in the department of Tacna still maintains outdated paradigms of
irrational resource use and overexploitation, manifesting itself in great
pressures and threats to the ecosystems, contrary to their conservation
objectives. For this reason, a meeting of experts (focus group) prioritized
the economic value of three ecosystem services that best represent their
importance: water resource provision, carbon sequestration and the
provision of genetic resources or livestock fodder. To determine the
economic value of these services, the revealed preferences and market
price method was applied, resulting in $\$ 1749548.65, \$ 454938.55$ and $\$$
1461388.35 respectively. These services were also valued based on their
development potential through the disclosed preferences method and on
avoided costs, market prices and production function, resulting in $\$ 3844$
$682.73, \$ 454938.55$ and $\$ 7254869.32$ respectively.

Ciencia \& Desarrollo, n. ${ }^{\circ} 27$, pp. 65-77 (2020) 


\section{INTRODUCCIÓN}

Ante la eminente pérdida de los ecosistemas y sus servicios esenciales para el bienestar humano a nivel mundial (Millennium Ecosystem Assessment, 2005), los países intentan protegerlos y conservarlos a través de sistemas nacionales de áreas naturales protegidas. De este modo, garantizar la provisión de bienes y servicios ecosistémicos materiales, regulatorios y culturales (Daily, 1997) que pueden ser valorados económicamente y que contribuyan a su conservación. En Perú, las áreas naturales protegidas se clasifican de acuerdo a su nivel de administración, siendo nacionales (ANP), privadas (ACP) y regionales o también llamadas Áreas de Conservación Regional (ACR) (Decreto Supremo No 016-2009-MINAM, 2009). En la zona altoandina del departamento de Tacna, al extremo sur del Perú, se encuentra el ACR Vilacota Maure, reconocido desde el 28 de agosto del 2009 (Decreto Supremo No015-2009, 2009), cuyo objetivo es la conservación de los ecosistemas de Puna de la cuenca del Maure para garantizar la constante y permanente provisión de los servicios ecosistémicos de los cuales depende el desarrollo del departamento de Tacna. Por ejemplo, del recurso hídrico necesario para el consumo humano, la agricultura y otras importantes actividades sociales y económicas. Sin embargo, al ser poco reconocido y valorado por la población, su gestión se encuentra permanentemente bajo presiones y amenazas que ponen en riesgo su conservación, como se evidencia en los diagnósticos de su plan maestro y en otras publicaciones (Navarro, 2015; Ordenanza Regional No 010-2018-CR/GOB.REG.TACNA, 2019).

La valoración económica es una valiosa herramienta para cuantificar, en términos monetarios, el valor de los bienes y servicios de los ecosistemas. Al calcular el valor económico de los servicios que brinda el ACR Vilacota Maure es posible garantizar su provisión futura (Ministerio del Ambiente, 2015; World Wildlife Fund, 2014), implementando mecanismos de retribución económica por parte de los usuarios de los servicios ecosistémicos evaluados (retribuyentes). En este sentido, es válido preguntarse ¿Cuánto es que el valor de los servicios ecosistémicos más representativos que provee el ACR Vilacota Maure puede contribuir en la mejora de la gestión y conservación?

A través de un Focus Group o reunión de expertos promovida por los autores de la presente investigación, se seleccionaron tres servicios ecosistémicos del ACR Vilacota Maure, priorizando aquellos que estén vinculados a alguna gran problemática regional o que satisfagan alguna necesidad poblacional de gran escala, asociada a alguna tendencia mundial o que satisfaga permanentemente alguna necesidad económica local. Los servicios elegidos para su valoración económica fueron la provisión de recurso hídrico, secuestro de carbono y provisión de forraje ganadero. Se espera que a partir de este conocimiento, se visibilicen todos aquellos beneficios o costos asociados a los cambios en los ecosistemas y de cómo algunas actividades humanas afectan el bienestar de la humanidad y de la sociedad.

\section{MATERIAL Y MÉTODOS}

\section{Área de Estudio}

El departamento de Tacna se ubica en el extremo sur del Perú, limita por el noroeste con el departamento de Moquegua, por el noreste con el departamento de Puno, por el sur con la República de Chile, por el este con la República de Bolivia y por el oeste con el Océano Pacífico. Tiene una superficie territorial de 16075.73 $\mathrm{km}^{2}$ que representa el $1.25 \%$ del país y está dividido en cuatro provincias: Tacna, Tarata, Candarave y Jorge Basadre (Ordenanza Regional No 001-2016-CR/GOB.REG.TACNA, 2016). El ACR Vilacota Maure tiene una superficie de $12.45 \mathrm{~km}^{2}$, representa el $7 \%$ del departamento y se encuentra entre los distritos de Palca, Tarata, Ticaco, Susapaya y Candarave (Decreto Supremo No015-2009-MINAM, 2009). Está formado por tres ecorregiones: Desierto de Sechura (56\%), Andes centrales (13\%) y Puna de los andes centrales (31\%), según el Centro de Datos para la Conservación de la Universidad Agraria de la Molina - CDC UNALM (2006) (Figura 1). 

del Perú

\section{Priorización de los servicios ecosistémicos}

En reunión de expertos (Focus Group) en conservación, biodiversidad, cultura, historia, guardaparques y especialistas, se discutió la problemática de los ecosistemas del ACR Vilacota Maure, así como las presiones y amenazas actuales, los servicios que otorgan y sus limitaciones para la provisión actual y futura. Luego, a través de un cuestionario de preguntas (Anexo 1), basado en las investigaciones de la United Kingdom National Ecosystem Assessment (2011), la Evaluación de Ecosistemas del Milenio para España (2011) y del Informe técnico de identificación de servicios ecosistémicos de Tacna (documento interno no publicado), se seleccionaron a tres servicios ecosistémicos prioritarios que fueron, según su criterio personal, experiencia técnica y profesional, los más importantes e influyentes para representar adecuadamente el ACR Vilacota Maure.

\section{Dimensionamiento}

La metodología para valoración económica de servicios ecosistémicos del ACR Vilacota Maure inicia con el dimensionamiento del bien y servicio ecosistémico localizando la zona de generación y el ámbito de influencia en el departamento, tanto para la población como para sus actividades productivas. Posteriormente, se estimó la capacidad de provisión del ACR Vilacota Maure. Con ello, se logró conocer y dimensionar el área territorial y la oferta de cada servicio ecosistémico.

\section{Categorización y Caracterización}

Para categorizar, tipificar y caracterizar cada servicio ecosistémico, fue necesario identificar los beneficios sociales, ambientales y económicos que genera el ACR Vilacota Maure y los beneficiarios directos, lo cual permitió seleccionar el adecuado método de valoración económica.

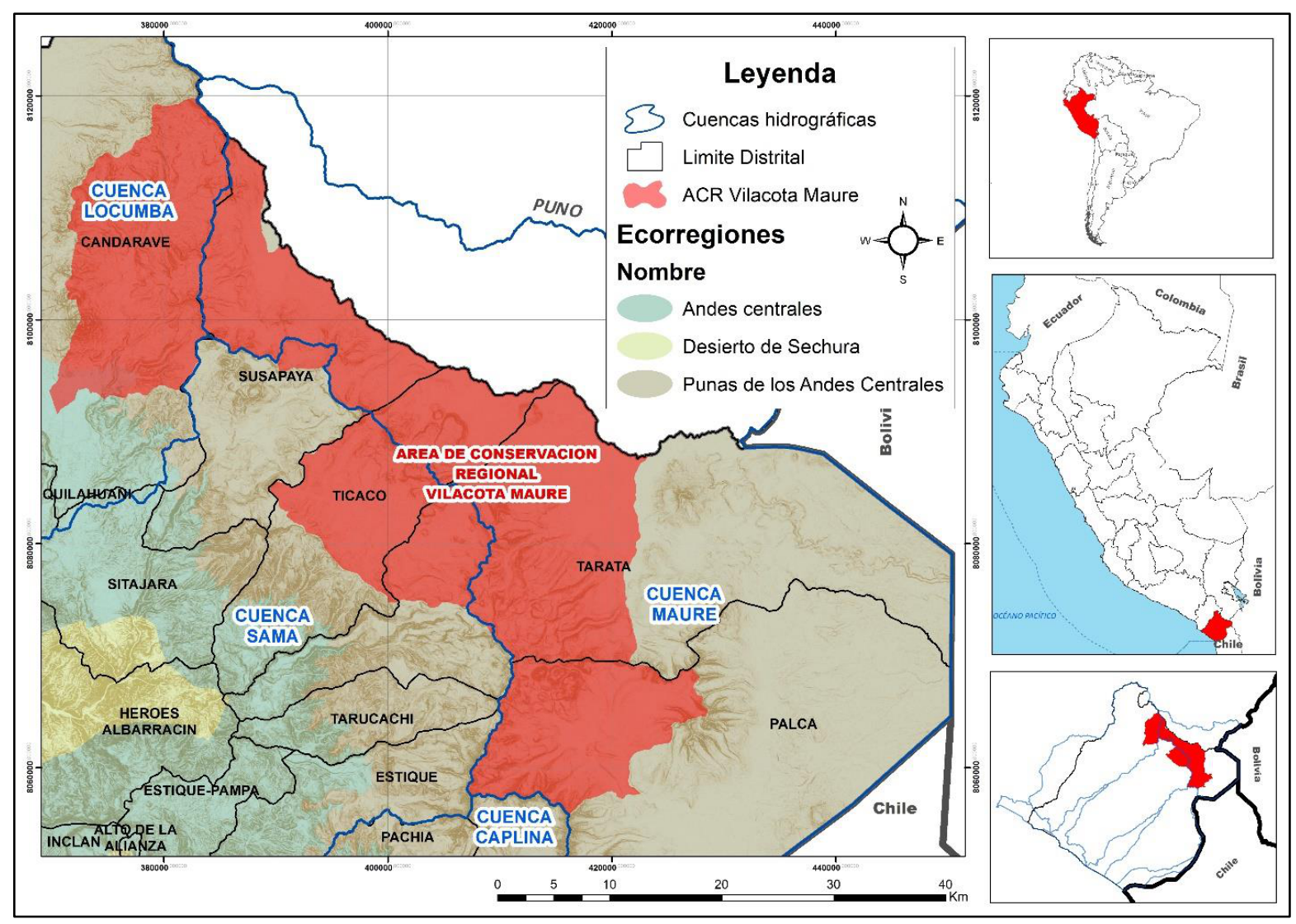

Figura 1. Área de estudio

Nota: Ubicación del Área de Conservación Regional Vilacota Maure en el departamento de Tacna donde se evidencia su distribución sobre la ecorregión Puna de los andes centrales. 


\section{Valoración Económica}

Los servicios ecosistémicos priorizados fueron valorizados económicamente bajo el criterio de valor de uso (es decir valorando los servicios que el ACR Vilacota Maure brinda en el momento presente y proyectado) y por el potencial de desarrollo (lo que puede producir si es que se mejora su capacidad productiva y si se realiza un cambio ambiental positivo). Para esto, se siguieron minuciosamente la secuencia de pasos descritos en la publicación del MINAM (2015), el cual está basado en los métodos de preferencia reveladas, los mismos que contemplan la recolección de información secundaria como insumos dentro del proceso de valoración. Solo para el caso de valoración económica del recurso genético o forraje ganadero, se tomó como referencia los valores de costos de una zona parecida al ACR, es decir, por comparación (Constanza et $a l, 1997)$ fue el departamento de Puno, en un área geográfica de desarrollo agrícola bajo condiciones y características muy similares al ACRVM (Provincia de Azángaro, Huancané y Melgar) y un producto similar o utilizado para los mismos fines como el cultivo de avena forrajera, utilizada para el pastoreo de los camélidos sudamericanos característicos también del ACR Vilacota Maure. De acuerdo al análisis realizado a este grupo control, se determinó el rendimiento de cultivo de avena forrajera por hectárea, el precio en chacra, los costos de producción, las ganancias y el ingreso.

Para la valoración económica de los 03 servicios ecosistémicos, se tomó como año base el 2018 y se emplearon parámetros dictados por el Ministerio de Economía y Finanzas (MEF) y el Ministerio del Ambiente (MINAM) a través de la Guía Nacional de Valoración Económica Del Patrimonio Natural, como un horizonte temporal de 22 años necesario para evaluar inversiones ambientales, fundamentado en la posibilidad de un cambio positivo ambiental, una tasa social de descuento de $5.50 \%$; procediendo así a obtener el Valor Presente Neto (VPN) mediante la fórmula del flujo descontado:

$$
\text { Valor Económico }=\frac{\mathrm{CF} 1}{(1+\mathrm{K})}+\frac{\mathrm{CF} 2}{(1+\mathrm{K}) 2}+\frac{\mathrm{CF} 3}{(1+\mathrm{K}) 3}+\ldots+\frac{\mathrm{CF}(\mathrm{n}+1)}{(\mathrm{k}-\mathrm{g})}
$$

El segundo miembro de la fórmula corresponde al valor a perpetuidad (VAP) que se calcula en base a los beneficios futuros descontándose la diferencia entre la tasa social de descuento ambiental y la tasa de mejora ambiental en el servicio ambiental gracias a inversiones ambientales (Huenchuleo et al., 2018).

\section{RESULTADOS}

Se determinó que el ACR Vilacota Maure provee a todo el departamento de Tacna veintitrés servicios ecosistémicos distribuidos en los cuatro grupos propuestos por la Millennium Ecosystem Assessment (2005), donde predominan los servicios ecosistémicos de regulación con nueve, seguido de los de provisión y culturales con cinco servicios cada uno y finalmente los servicios de soporte con cuatro, cuyos tipos, grupos, enfoques y métodos de valoración se discutieron en la reunión de expertos, proponiéndose la lista que se presenta en la Tabla 2, en la que se observa que es posible valorar económicamente todos los servicios ecosistémicos del ACR Vilacota Maure, mayoritariamente por los métodos de preferencias reveladas (20) mientras que muy pocas (3) por los de preferencias indicadas.

Los servicios ecosistémicos priorizados por el grupo de expertos fueron principalmente los que se encuentran altamente relacionados con la problemática regional puesta de manifiesto de forma verbal en la reunión de expertos, pero a la vez sustentada y documentada por normas y leyes, siendo los seleccionados el servicio de provisión de recurso hídrico o agua para consumo humano (basado en el Decreto Supremo $\mathrm{N}^{\circ}$ 004-2009-AG, 2009), recursos genéticos o forraje ganadero (basado en estadísticas de pobreza del INEI, 2018) y secuestro de carbono (basado en la Estrategia Regional de Cambio Climático de Tacna según Ordenanza Regional No 005-2015-CR/GOB.REG.TACNA, 2015). 


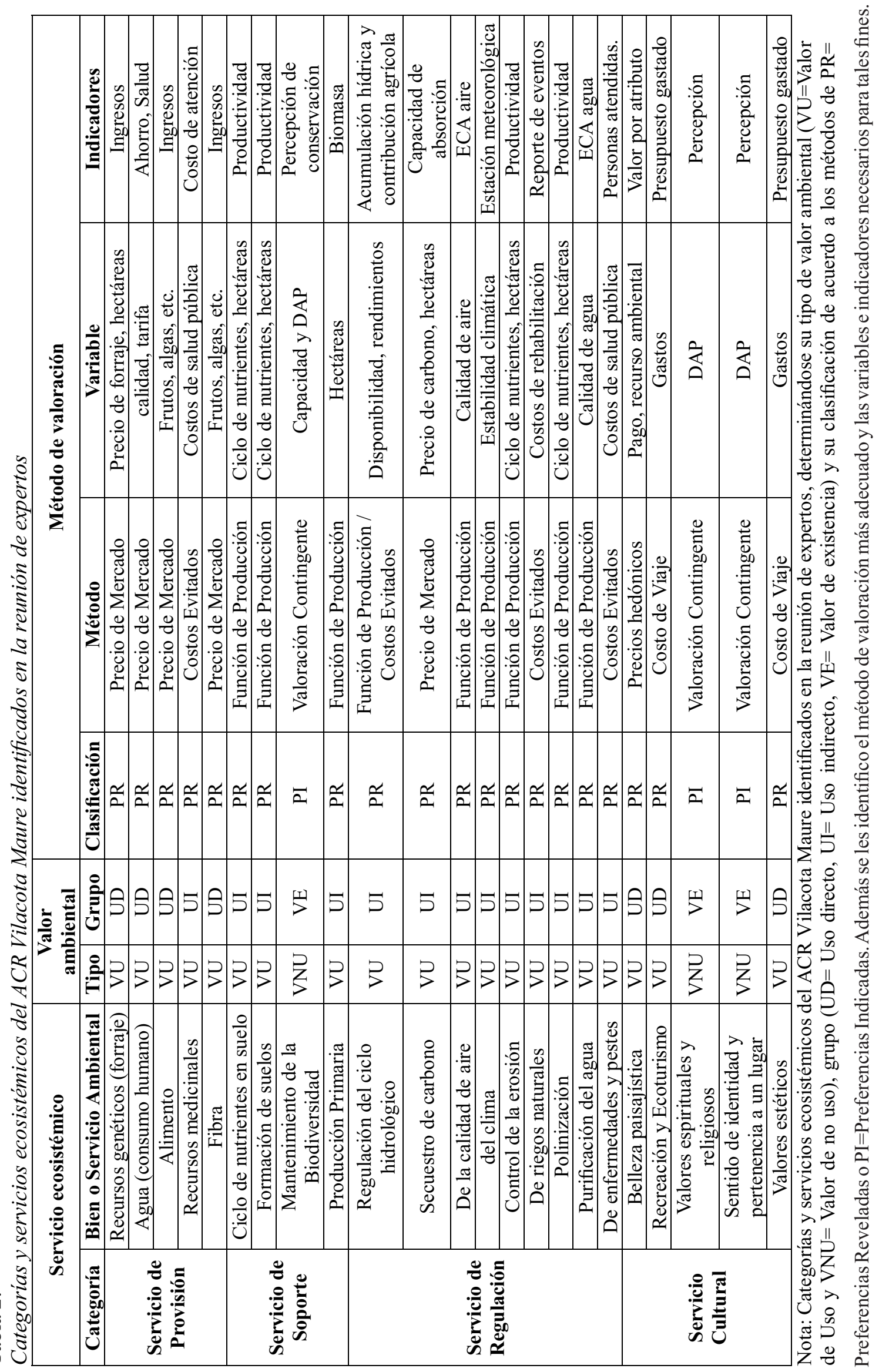




\section{Dimensionamiento}

El caudal ecológico del ACR Vilacota Maure es de 209.7 millones de metros cúbicos anuales, considerándose un caudal entre agua de manantial más la fuente termal un total de $113.6 \mathrm{Mm}^{3}$, el río Maure con $36 \mathrm{Mm}^{3}$, canales con $3.1 \mathrm{Mm}^{3}$, el túnel de Kovire con $12 \mathrm{Mm}^{3}$, presas de $32 \mathrm{Mm}^{3}$, lagunas de $13 \mathrm{Mm}^{3}$, haciendo un total de caudales de $209.7 \mathrm{Mm}^{3}$, pero que sufre pérdidas de agua de forma natural por filtración, evaporación, y sustracción. Todo ello asciende al $30 \%$ del stock inicial, por lo cual la oferta hídrica neta asciende a 146.79 millones de metros cúbicos anuales; asimismo, la disponibilidad de agua planificada y programada por el ANA para el consumo humano es de $3.7 \%$; por tanto, corresponde para consumo humano 5.4 millones de metros cúbicos anualmente.

El ACR Vilacota Maure tiene una superficie de 124234.83 hectáreas de las cuales actualmente se utilizan 208.67 hectáreas para agricultura y 1636.26 hectáreas para forestación, es decir, solo el $1.5 \%$ de su territorio, las cuales tienen un rendimiento promedio anual de 20000 kilogramos de forraje por hectárea cosechada. Por tanto, el ACR Vilacota Maure provee de 4173.40 toneladas de forraje anual para la ganadería en camélidos sudamericanos.

Una hectárea del ACR Vilacota Maure tiene la capacidad de secuestro de 0.88 toneladas de carbono, comprendiendo un área de bosques de queñuales de 3895.84 hectáreas, su capacidad actual de retención es de 3428.34 toneladas de carbono anuales, contribuyendo así al combate frente al calentamiento global y la contaminación ambiental.

\section{Categorización y caracterización}

El recurso hídrico para consumo humano es un servicio ecosistémico de la categoría de provisión, su tipo de valor ambiental es de valor de uso y pertenece al grupo de uso directo. El método de valoración clasifica como preferencias reveladas bajo el método de precios de mercado, y las variables de estudio son el caudal ecológico, la distribución y disponibilidad del recurso hídrico para el consumo humano, la demanda poblacional en la ciudad de Tacna y la retribución económica por el servicio. El beneficio social es la satisfacción del consumo humano y salud.

El recurso natural genético es un servicio ecosistémico de provisión y su tipo de valor ambiental es el valor de uso perteneciente al grupo de uso directo; el método de valoración económica clasifica como preferencias reveladas con el método de precios de mercado, y las variables de estudio son el área disponible para agricultura en el ACR Vilacota Maure, la producción de forraje por hectárea por cosecha

El secuestro de carbono es un servicio ecosistémico en la categoría de regulación, su tipo es valor de uso en el grupo de uso indirecto; respecto al método de valoración clasifica como preferencia reveladas bajo el método de precio de mercado, en este caso se aplica el precio social del carbono y la capacidad de absorción por hectárea del ACR Vilacota Maure.

\section{Valoración económica}

En la Tabla 3 se presenta de forma detallada las variables e indicadores utilizados durante el procedimiento de valoración económica aplicado a los servicios ecosistémicos priorizados en el que también se presentan los valores encontrados en diferente bibliografía revisada como el Plan de Gestión de los Recursos Hídricos (Autoridad Nacional del Agua, 2015), el Plan Basadre (Ordenanza Regional N 001-2016CR/GOB.REG.TACNA, 2016)

El servicio ecosistémico de provisión de recurso hídrico tiene un valor presente neto de S/ 2432978.37 y un valor a perpetuidad de S/ 3515 487.05; por lo cual el valor económico total del servicio ecosistémico que provee el ACR Vilacota Maure asciende a S/ 5948465.42. 
Valoración económica del Área de Conservación Regional Vilacota Maure: Servicios ecosistémicos priorizados al extremo sur del Perú

Tabla 3

Variables e indicadores utilizados para calcular el valor económico de los servicios ecosistémicos priorizados del ACR Vilacota Maure según documentación técnica en población, salud, gestión de recursos hídricos entre otras

\begin{tabular}{|c|c|c|}
\hline $\begin{array}{c}\text { Servicio } \\
\text { Ecosistémico }\end{array}$ & Variables e Indicadores & Valor \\
\hline \multirow{12}{*}{ 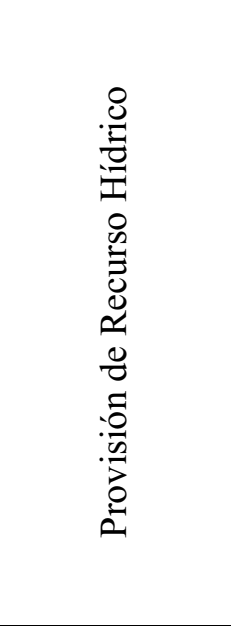 } & Caudal ecológico ACRVM (millones $\mathrm{m}^{3}$ ) & 209700000.00 \\
\hline & Pérdida de recurso hídrico $(\%)$ & 0.30 \\
\hline & Recurso hídrico disponible & 146790000.00 \\
\hline & Destino para consumo humano & 0.04 \\
\hline & Disponible consumo humano & 5431230.00 \\
\hline & Horizonte de evaluación (años) & 22.00 \\
\hline & Tasa de descuento ambiental & 0.06 \\
\hline & Retribución económica por metro cúbico & 0.04 \\
\hline & Tasa de mejora ambiental & 0.03 \\
\hline & Mejora de eficiencia y uso hídrico & 0.90 \\
\hline & Potencial por mejora ambiental & 188730000.00 \\
\hline & Potencial disponible para consumo & 6983010.00 \\
\hline \multirow{8}{*}{ 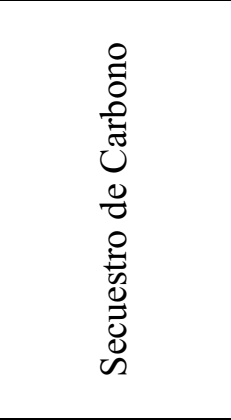 } & Superficie & 3895.84 \\
\hline & T.M. X Ha. & 0.88 \\
\hline & Capacidad actual & 3428.34 \\
\hline & Precio X T.M. & 23.66 \\
\hline & Ingresos & 81114.51 \\
\hline & Tasa de crecimiento & 0.03 \\
\hline & Tasa de descuento & 0.06 \\
\hline & Periodo de evaluación & 22.00 \\
\hline \multirow{12}{*}{ 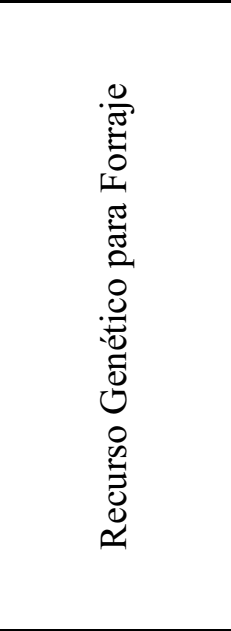 } & Área agrícola actual & 208.67 \\
\hline & Área potencial & 7867.67 \\
\hline & Área factible & 786.77 \\
\hline & Productividad & 20000.00 \\
\hline & Precio & 0.30 \\
\hline & Costo de producción & 4978.50 \\
\hline & Costo unitario & 0.25 \\
\hline & Tasa de descuento & 0.06 \\
\hline & Horizonte temporal & 23.00 \\
\hline & Cosecha inicial & 208.67 \\
\hline & Tasa de crecimiento ambiental & 0.03 \\
\hline & Tasa de incremento de área productiva & 0.00 \\
\hline
\end{tabular}

Nota: Elaboración propia.

Fuente: Basado en INEI(2018); ANA, AgroPuno (2017) (No publicada), INIA(2007) (tríptico no publicado).

Dicho servicio ecosistémico tiene un potencial de desarrollo basado en la disminución de la pérdida natural con lo cual se incrementaría la disponibilidad del recurso hídrico y su valor se incrementaría a S/10 167 049.89 . 
El servicio ecosistémico de provisión de recurso genético para forraje que permite la alimentación de camélidos sudamericanos en ACR Vilacota Maure tiene un valor presente neto de S/ 2480139.24 y un valor a perpetuidad de $\mathrm{S} / 2488581.15$, por lo cual el valor económico total de dicho servicio ecosistémico es de $\mathrm{S} /$ 4968720.38 .

El recurso genético tiene un potencial de desarrollo basado en la disponibilidad de área para uso agrícola de 7867.67 hectáreas; por lo cual, asumiendo una factibilidad del $10 \%$, tendría un incremento de 787 hectáreas. Por lo tanto, el valor económico potencial alcanzaría los S/24 666 555.67.

El servicio ecosistémico de secuestro de carbono tiene un valor presente neto de S/ 1020677.59 y un valor a perpetuidad de S/ 526 113.49; por tanto, el valor económico total del servicio ecosistémico es de S/ 1546 791.08, considerando una tasa de mejora ambiental del $1 \%$.

Es así que en la Tabla 4 se presentan los resultados obtenidos del valor económico calculado (en dólares americanos) bajo el enfoque de valor de uso. El valor económico total de los 03 servicios ecosistémicos que provee el ACR Vilacota Maure asciende a la suma de S/ 12463976.89 o su equivalente de U\$ 3665875.55 dólares (al tipo de cambio de $\mathrm{S} / 3.4$ por dólar).

\section{Tabla 4}

Valores económicos de los servicios ecosistémicos priorizados calculado bajo los enfoques de valor de uso ${ }^{l}$ y de producción de desarrollo en el que se considera tambien el Valor Presente Neto (VPN) y el Valor a Perpetuidad (VaP)

\begin{tabular}{|c|c|c|c|}
\hline \multicolumn{4}{|c|}{$\begin{array}{l}\text { 1. Valoración económica de los Servicios Ecosistémicos por Valor de uso (en dólares } \\
\text { americanos) }\end{array}$} \\
\hline $\begin{array}{c}\text { Servicio } \\
\text { ecosistémico }\end{array}$ & $\begin{array}{l}\text { Provisión de recurso } \\
\text { hídrico }\end{array}$ & Recursos genéticos & Secuestro de carbono \\
\hline Método & $\begin{array}{l}\text { Preferencias reveladas - } \\
\text { Precios de Mercado }\end{array}$ & $\begin{array}{c}\text { Preferencias } \\
\text { Reveladas - Precios } \\
\text { de Mercado }\end{array}$ & $\begin{array}{l}\text { Preferencias Reveladas - } \\
\text { Precios de Mercado }\end{array}$ \\
\hline VPN & $\$ 715581.87$ & $\$ 729452.72$ & $\$ 300199.29$ \\
\hline VaP & $\$ 1033966.78$ & $\$ 731935.63$ & $\$ 154739.26$ \\
\hline Valor económico & $\$ 1749548.65$ & $\$ 1461388.35$ & $\$ 454938.55$ \\
\hline \multicolumn{4}{|c|}{$\begin{array}{l}\text { 2. Valoración económica de los Servicios Ecosistémicos por Potencial de desarrollo (en } \\
\text { dólares americanos) }\end{array}$} \\
\hline $\begin{array}{c}\text { Servicio } \\
\text { ecosistémico }\end{array}$ & $\begin{array}{l}\text { Provisión de recurso } \\
\text { hídrico }\end{array}$ & Recursos genéticos & Secuestro de carbono \\
\hline Método & $\begin{array}{c}\text { Preferencias reveladas- } \\
\text { Disminución de pérdida } \\
\text { natural de agua }\end{array}$ & $\begin{array}{c}\text { Preferencias } \\
\text { Reveladas - Función } \\
\text { de Producción - } \\
\text { Ampliación de área } \\
\text { agrícola } \\
\end{array}$ & $\begin{array}{l}\text { Preferencias Reveladas - } \\
\quad \text { Precios de Mercado }\end{array}$ \\
\hline $\begin{array}{l}\text { Beneficio Social } \\
\text { por costo evitado/ } \\
\text { inversión }\end{array}$ & $\$ 3844682.73$ & $\$ 7254869.32$ & $\$ 454938.55$ \\
\hline
\end{tabular}


Los servicios ecosistémicos de provisión de recurso hídrico, recurso genético para forraje y secuestro de carbono tienen un potencial de desarrollo conjunto con un valor económico ascendente a S/ 39285264.04 o su equivalente a U\$\$11 554490.60 .

\section{DISCUSIÓN}

Medina (2019) obtuvo un valor económico total de USD 119034797.58 (ciento diecinueve millones treinta y cuatro mil setecientos noventa y siete dólares) para algunos servicios de la la Reserva Nacional de Salinas y Aguada blanca, siendo el servicio de secuestro y almacenamiento de $\mathrm{CO}_{2}$ el que aportó el $81.36 \%$ de dicho valor, seguido del servicio de ecoturismo (18.32\%) y del bien Fibra de vicuña (0.32 \%). Asimismo, Loyola (2007) estimó que los recursos hídricos de la reserva tienen un valor de 2132502.57 (dos millones ciento treinta dos mil quinientos dos dólares), relativamente más bajo para lo calculado para el ACR Vilacota Maure, considerando que se ubican en la misma ecorregión, la Puna de los Andes Centrales.

Es así que el principal problema identificado por los expertos reunidos en el focus group y servicio de provisión de recurso hídrico, cuya mayor justificación se encontró en el Decreto Supremo Nº04-2009-AG (2009), el cual declara agotados los recursos hídricos superficiales de las cuencas de los ríos Caplina, Sama y Locumba, es decir, en tres de las cuatro cuencas del departamento, por lo que, desde hace algunos años atrás y a través de diferentes proyectos de afianzamiento hídrico se produce el trasvase de agua de la cuenca del Maure (Vilavilani I) hacia las de Sama y Caplina para diversos fines (poblacional, agrícola, electrificación, entre otros). Asimismo, existen en cartera y priorizados en el Plan Regional de Desarrollo Concertado (Ordenanza Regional No 001-2016-CR/GOB.REG.TACNA, 2016) futuros proyectos de trasvase de agua de la cuenca del Maure como el Proyecto Vilavilani II, Embalse Calientes Santa Cruz, Embalse Jarumas II, Canal Chiquitoma, entre otros, por lo que se vuelve fundamental que estos se desarrollen sin que pongan en riesgo la conservación de los importantes ecosistemas que se encuentran al interior del ACR Vilacota Maure.

Asimismo, con la emisión de la Ley No 30215 y su reglamento (Decreto Supremo No 009-2016-MINAM, 2016) se establece la posibilidad de que las empresas prestadoras de servicio de agua potable (EPS), establecidas a nivel nacional, establezcan mecanismos de retribución por servicio ecosistémico hídrico, para que a través de cobros por tarifas, los retribuyentes (usuarios) aporten dinero para el financiamiento de proyectos que mejoren y mantengan el estado de conservación a favor de los ecosistemas, considerando la presencia de contribuyentes (como el ACR VM) para la conservación de los ecosistemas. Este mecanismo requerirá cálculos que pueden ser los obtenidos de la presente investigación.

Los proyectos de conservación deberían basarse en experiencias exitosas de manejo sustentable del territorio, como lo planificado en el proyecto que el Global Environment Fund (GEF) del Banco Mundial viene ejecutando con la Corporación Nacional Forestal - CONAF de la República de Chile (Aguilera, 2012), donde se prioriza la reforestación y el manejo de bofedales que mejoren el riego de las pasturas para las actividades económicas locales y para garantizar la provisión de los servicios ecosistémicos como el forraje ganadero.

En Chile y Perú se vienen haciendo grandes esfuerzos por valorar económicamente el patrimonio natural, tanto fuera de las ANP como al interior de ellas a través de sus respectivos sistemas nacionales de áreas protegidas, para relacionarlos con las condiciones del bienestar humano y demostrar la importancia y necesidad de conservar los ecosistemas naturales. A razón de esto se han realizado trabajos articulados y llevado a cabo diversos proyectos, evidenciados en informes técnicos, guías y manuales que vienen orientando la gestión territorial de estos países vecinos y hermanos (Figueroa, 2010; Salgado et al., 2015)

Se señala que es muy importante conocer el valor económico de los bienes y servicios ecosistémicos porque ayuda a los administradores a mejorar la gestión de las ANP y sus ecosistemas naturales; asimismo, fortalece a los Gobiernos para definir políticas claras respecto a la conservación mediante nuevos 
lineamientos, en búsqueda de la sostenibilidad financiera a partir de la comercialización de bienes y SE mediante tarifas, compensaciones, pagos por servicios ambientales y mecanismos de retribución de servicio ecosistémico (Ministerio del Ambiente, 2016)

\section{CONCLUSIONES}

Se ha determinado el valor económico de tres servicios ecosistémicos del ACR Vilacota Maure, priorizados de forma participativa por su representatividad o por su importancia para el bienestar humano. Así, el alto valor económico obtenido por el servicio de provisión del recurso hídrico representa una excepcional oportunidad para implementar mecanismos de conservación asociado a los de retribución por servicio ecosistémico, evidenciándose y resaltándose la importancia de conservar los ecosistemas altoandinos del extremo sur del Perú, Chile y Bolivia.

Los tres servicios ecosistémicos valorados tienen un alto potencial de desarrollo económico que aliviarían problemas de desarrollo, generando bienestar en la región Tacna. Por tanto, se hace necesario contar con un programa de inversión ambiental y un mecanismo de financiamiento autosostenible para garantizar su sostenibilidad y conservación de la provisión de servicios ecosistémicos del ACR Vilacota Maure.

A pesar que existen muchos vacíos de información respecto a la capacidad de los ecosistemas altoandinos para fijar carbono, resulta muy importante la conservación de los ecosistemas del ACR Vilacota Maure al considerarlos como sumideros de carbono, cuyo beneficio en la regulación del clima es evidente y ahora cuantificable.

Es necesaria la gestión de conocimiento para lograr la valoración económica de los 20 servicios ecosistémicos que provee el ACR Vilacota Maure que no han sido evaluados y valorados por esta investigación. Además, se ha determinado su importancia como reserva alimenticia para los camélidos sudamericanos silvestres (vicuñas y guanacos) y domésticos (llama y alpaca), lo que sustenta una cantidad importante en la economía local, cuya tasa de retorno puede ser muy valiosa para sustentar programas de inversiones que mejoren la calidad ganadera del ámbito.

Se espera que la información obtenida ayude a la gestión del ACR Vilacota Maure y se encamine en un modelo de gestión territorial importante que sea replicable en otras zonas con similares ecosistemas y servicios, respetándose la conservación de estos por todos sus valores, los estéticos, ecológicos, culturales, paisajísticos u otros, pero también por su valor económico, reflejado en los resultados de la presente investigación que se espera sea de gran utilidad.

\section{REFERENCIAS}

Aguilera, G. (2012). Evaluación social: Proyecto manejo sustentable de la tierra. Corporación Nacional Forestal-CONAF. 161p. Disponible en http://www.conaf.cl/cms/editorweb/GEF-BM/EvaluacionSocial-PROYECTO_GEF-BM.pdf

Autoridad Nacional del Agua. (2015). Plan de gestión de los recursos hídricos de la cuenca Caplina Locumba. Lima, Perú: Autoridad Nacional del Agua. Proyecto de Modernización de la Gestión de los Recursos Hídricos. 204p. http://repositorio.ana.gob.pe/handle/20.500.12543/88

CDC-UNALM. (2006). Análisis del Recubrimiento Ecológico del Sistema Nacional de Áreas Naturales Protegidas por el Estado. Lima: CDC-UNALM/TNC. 170p. Disponible en http://cdc.lamolina.edu.pe/Descargas/ANPs/PlanDirector.html

Costanza, R., dÁrge, R., de Groot, R., Farber, S., Grasso, M., Hannon, B., Limburg, K., Naeem, S., Oneill, R., Paruelo, J., Raskin, R., Sutton, P., \& Van Den Belt, M., (1997). The value of the world's ecosystem services and natural capital. Nature 387, 253-260. https://doi.org/10.1038/387253a0 
Daily, G. (1997). Nature's Services: Societal Dependence on Natural Ecosystems. Washington, DC: Island Press. 392 p.

Decreto Supremo No 004-2009-AG. (2009). Declaran agotados los recursos hídricos superficiales de las cuencas de los ríos Caplina, Sama y Locumba. Diario Oficial El Peruano, Lima, Perú, 08 de febrero $\begin{array}{lllll}\mathrm{d} \text { e } 1 & 2009 & \mathrm{R} \text { e c u p e r a d o } & \mathrm{d} \mathrm{e}\end{array}$ http://minagri.gob.pe/portal/download/pdf/marcolegal/normaslegales/decretossupremos/ds_004_ 2009_ag.pdf

Decreto Supremo No 015-2009-MINAM, (2009) Se establece el Área de Conservación Regional Vilacota Maure y se desafecta la Zona Reservada Aymara Lupaca. Diario Oficial El Peruano. Lima, Perú, 28

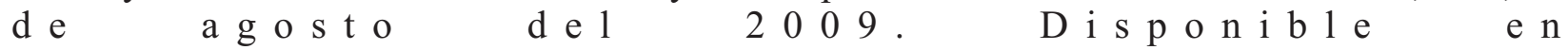
http://www.gacetajuridica.com.pe/servicios/normaspdf_2009/Agosto/28-08-2009.pdf

Decreto Supremo No 016-2009-MINAM. (2009) Plan Director de las Áreas naturales Protegidas. Diario Oficial El Peruano. Lima, Perú, 03 de setiembre del 2009. Disponible en https://busquedas.elperuano.pe/normaslegales/decreto-supremo-que-establece-el-area-deconservacion-region-decreto-supremo-n-008-2019-minam-1812452-7/

Decreto Supremo No 009-2016-MINAM (2016) Aprueban Reglamento de la Ley No 30215, Ley de Mecanismos de Retribución por Servicios Ecosistémicos. Diario Oficial El Peruano, Lima, Perú, 21 de julio del 2016. https://busquedas.elperuano.pe/normaslegales/aprueban-reglamento-de-la-ley-n30215-ley-de-mecanismos-de-decreto-supremo-n-009-2016-minam-1407244-4/

Evaluación de ecosistemas del milenio de España. (2011). Informe de síntesis EME 2012 [online] Disponible en: http://www.ecomilenio.es/informe-sintesis-eme/232

Figueroa, E. (2010) Valoración Económica detallada de las áreas protegidas de chile. Santiago, Chile. $\mathrm{S}$ a 1 e s i a n o s I m p e s o r e s S. A. $233 \mathrm{p}$. D i s p o n i b 1 e e $n$ http://bdrnap.mma.gob.cl/recursos/privados/Recursos/CNAP/GEF-SNAP/Figueroa_2010.pdf

Huenchuleo, C., \& de Kartzow, A. (2018). Valoración económica de servicios ecosistémicos en la cuenca del río Aconcagua, Chile. Tecnología y Ciencias del Agua, 09(2), 58-85. https://doi.org/10.24850/jtyca-2018-02-03

INEI. (2018). Perú: Perfil Sociodemográfico. Informe Nacional. Instituto Nacional de Estadística e

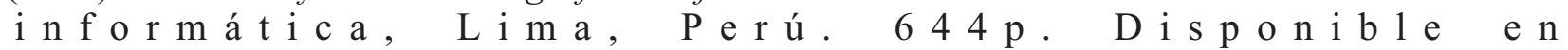
https://www.inei.gob.pe/media/MenuRecursivo/publicaciones_digitales/Est/Lib1539/libro.pdf

Loyola, R. (2007). Valoración del Servicio Ambiental de Provisión de Agua con Base en la Reserva Nacional Salinas y Aguada Blanca - Cuenca del Río Chili. PROFONANPE. Lima. 228 p.

Medina, C. (2019). Valoración económica de la diversidad biológica en la Reserva Nacional Salinas y Aguada Blanca - Arequipa, Perú (Tesis presentada el Grado académico de Maestro en Ciencias: con mención en seguridad y medio ambiente). Universidad Nacional San Agustín. Disponible en http://repositorio.unsa.edu.pe/handle/UNSA/9849

Millennium Ecosystem Assessment (2005). A Report of the Millennium Ecosystem Assessment. Ecosystems a n d H u m n W 11 - b e i g. D i s o n i b e e n : http://www.millenniumassessment.org/documents/document.356.aspx.pdf

Ministerio del Ambiente (2015). Guía nacional de valoración económica del patrimonio natural. Lima, Perú. Disponible en http://www.minam.gob.pe/patrimonio-natural/wpcontent/uploads/sites/6/2013/10/GVEPN-30-05-16-baja.pdf

Ministerio del Ambiente (2016). Guía complementaria para la compensación ambiental de los ecosistemas altoandinos. Lima, Perú: MINAM. Disponible en http://goo.g1/FVar8r 
Navarro, M. (2015). El Reto de la Conservación en Tacna. Tacna, Perú: SERNANP. 100p. https://www.researchgate.net/publication/305318922_El_Reto_de_la_Conservacion_en_Tacna

Ordenanza Regional No 005-2015-CR/GOB.REG.TACNA. (2015). Aprueban el instrumento de Gestión Ambiental Regional denominado: "Estrategia Regional de Cambio Climático (ERCC) de Tacna" y dictan otras disposiciones. Diario Oficial El Peruano, Lima, Perú, 14 de enero del 2015. Disponible en https://busquedas.elperuano.pe/normaslegales/aprueban-el-instrumento-de-gestion-ambientalregional-denomi-ordenanza-no-005-2015-crgobregtacna-1273301-1/

Ordenanza Regional No 001-2016-CR/GOB.REG.TACNA. (2016). Aprueban el Plan de Desarrollo Regional Concertado Tacna hacia el 2021. Diario Oficial El Peruano, Lima, Perú, 22 de Abril del 2016. https://busquedas.elperuano.pe/download/url/aprueban-el-plan-de-desarrollo-regionalconcertado-tacna-h-ordenanza-no-001-2016-crgobregtacna-1370266-2

Ordenanza Regional No 010-2018-CR/GOB.REG.TACNA (2019). Aprueban Plan Maestro 2017-2021 del Área de Conservación Regional Vilacota Maure. Diario Oficial El Peruano. Lima, Perú, 13 de marzo del 2019. Disponible en https://vlex.com.pe/vid/772175421

Salgado, H., De la Puente, S., González, C., \& Sueiro, J. (2015). Valoración Económica Total (VET) de los bienes y servicios ecosistémicos del Gran Ecosistema Marino de la Corriente de Humboldt $(G E M C H)$. Consultoría elaborada para el Proyecto GEF-PNUD: Hacia un manejo con enfoque ecosistémico de Gran Ecosistema Marino de la Corriente de Humboldt. Talca, Chile / Lima, Perú. $102 \mathrm{p}$.

United Kingdom National Ecosystem Assessment, 2011. Technical Report 2011 [online] Disponible en: http://uknea.unep-wcmc.org/

World Wildlife Fund (2014). Guía práctica para la valoración económica de servicios ecosistémicos en Madre de Dios. Editorial Calambur. Lima, Perú. 
Anexo 1.

\section{Cuestionario utilizado en el Focus Group}

VALORACIÓN ECONÓMICA DEL ACRVM

\section{ENTREVISTA DE LEVANTAMIENTO DE INFORMACIÓN TÉCNICA}

\section{INFORMACIÓN GENERAL}

Consideras importante un estudio de Valoración Económica del ACRVM *

○ Sí

- No

Sustenta tu respuesta *

\section{PROBLEMÁTICA DEL ACRVM}

Todo proceso de valoración económica se inicia con la identificación de un problema referido a la falta de un proyecto, un programa, una norma o una politica que permite cambios en los ecosistemas o en los servicios que ellos nos brindan. Te pedimos que compartas con nosotros 5 problemas que hayas identificado en el desarrollo de tus funciones, que incluya la alternativa de solución.

\section{PRESIONES Y AMENAZAS DEL ACRVM}

Por presiones se entienden las acciones de origen humano que ponen en peligro la integridad de los ecosistemas y los derechos colectivos de sus habitantes. Las amenazas, de otro lado, son iniciativas previstas para el futuro próximo que pueden convertirse en presiones una vez implementadas.

\section{SERVICIOS ECOSISTEMICOS DEL ACRVM}

Son los beneficios que los ecosistemas otorgan a las personas.

Qué servicio de soporte consideras el más importante del ACRVM

- Formación del suelo.

○ Ciclo de nutrientes.

- Producción primaria.

- Mantenimiento de la biodiversidad.

Qué servicio de regulación consideras el más importante del ACRVM *

- Regulación del clima

- Purificación del agua y tratamiento de aguas de desecho

- Regulación hídrica

- Regulación de riesgos naturales

- Regulación de enfermedades y pestes

- Regulación de la calidad de aire

- Control de la erosión

- Polinización

- Secuestro de carbono

Qué servicio cultural consideras el más importante del ACRVM*

- Belleza paisajística

- Valores espirituales y religiosos

- Valores estéticos

- Recreación y ecoturismo

- Sentido de identidad y pertenencia a un lugar

Qué servicio de provisión consideras el más importante del ACRVM *
○ Agua
- Alimento
- Recursos genéticos
- Recursos medicinales
- Fibra 